\title{
Alternating-code experiment for plasma-line studies
}

\author{
P. Guio ${ }^{1}$, N. Bjørnå ${ }^{1}$, W. Kofman ${ }^{2}$ \\ 1 The Auroral Observatory, University of Tromsø, Tromsø, N-9037 Norway \\ 2 CEPHAG Domaine Universitaire, BP 46, St-Martin-D'hères, F-38402 France
}

Received: 15 January 1996/Revised: 30 April 1996/Accepted: 2 May 1996

\begin{abstract}
We present results of the first plasma-line measurement of the incoherent spectrum using the alternating-code technique with the EISCAT VHF radar. This technique, which has earlier mostly been used to measure high-resolution E-region ion-line spectra, turned out to be a very good alternative to other techniques for plasmaline measurements. The experiment provides simultaneous measurement of the ion line and downshifted and upshifted plasma-line spectra with an altitude resolution of $3 \mathrm{~km}$ and a temporal resolution of $10 \mathrm{~s}$. The measurements are taken around the peak of the $\mathrm{F}$ region, but not necessarily at the peak itself, as is the case with the longpulse technique. The condition for success is that the scale height should be large enough such that the backscattered signal from the range extent of one gate falls inside the receiver filter. The data are analyzed and the results are combined with the results of the ion-line data analysis to estimate electron mean drift velocity and thereafter electric currents along the line of sight of the radar using both the standard dispersion relation assuming a Maxwellian electron velocity distribution and the more recent model including a heat-flow correction term.
\end{abstract}

\section{Introduction}

The incoherent scattering spectrum consists of two main components. The ion line, which contains most of the scattered power, is extensively being measured at the different facilities around the world, and standard parameters such as electron density, electron and ion temperature, ion mean drift velocity, and others are diagnosed. On the other hand, plasma lines are weak and broadband. The intrinsic spectral width of the plasma line is determined by the damping of the Langmuir wave causing the scattering (Landau damping or collisional damping). The damping depends on the density, temperature and collision frequency of the ambient electrons and on the presence of a suprathermal population (photoelectrons or secondary electrons produced in particle precipitation events) around the phase velocity of the wave (Perkins and Salpeter, 1965). It also depends on the angle between the scattering direction and the geomagnetic field. For small angles, as in this experiment, the effect is negligible. In the $F$ region at daytime, where damping is mostly dominated by electron-ion collisions, and for field-aligned conditions, the width is a few $\mathrm{kHz}$. Temporal fluctuations during the integration time artificially broaden the width. Under relatively stable conditions of the ionosphere, this broadening is a few $\mathrm{kHz}$ per $10 \mathrm{~s}$, which is a typical integration time. The spatial variation depends on the electron-density scale height which is of the order of $1 \mathrm{kHz} \mathrm{km}^{-1}$ at regions with large scale height and around the peaks in the $\mathrm{E}$ and $\mathrm{F}$ regions up to $10 \mathrm{kHz} \mathrm{km}^{-1}$. The scattered power is usually less than $10 \%$ of the ionline power, even in the presence of suprathermal particles. This leads to some conditions on the measurement of the autocorrelation function. The correlation time is inversely proportional to the bandwidth of the plasma line and is not larger than $500 \mu \mathrm{s}$. The lag increment $\tau$ determines the bandwidth of the measured spectra and should be around $10-20 \mu$ s for a spectral window of $50-100 \mathrm{kHz}$.

To perform the measurement of the autocorrelation function several techniques are available and can be classified into three categories. The long-pulse technique (Showen, 1979; Kofman and Wickwar, 1980) provides data at the cut frequency of the $\mathrm{E}$ or $\mathrm{F}$ region. It has the advantage of a good signal-to-noise ratio, which increases with increasing density scale height. On the other hand, this technique provides only one measurement point. The chirp technique (Hagfors, 1982; Birkmayer and Hagfors, $1986)$ is very similar to the long-pulse technique. The signal is chirped in order to tune it to the local density gradient, creating artificially the measurement conditions of a peak density in a region. In these techniques, no attempt is made to obtain good range resolution. 
The multipulse technique (Farley, 1972), by using ad hoc spatial patterns of short pulses, provides good range resolution and good lag resolution. Unfortunately, the multiple-frequency technique commonly used to improve the accuracy of the measurement of the ion line is not adequate for plasma-line measurements due to the $k$ dependence of the position of the plasma line which would broaden the spectra. In low signal-to-noise ratio measurements, like plasma-line measurements, one definitely needs to concentrate all of the energy on the same frequency. Also the accuracy of position estimation will be reduced unless special analysis techniques are used to account for it (using a 4-frequency multipulse would yield a broadening of $\sim 150 \mathrm{~Hz}$ with the EISCAT VHF radar and $\sim 1 \mathrm{kHz}$ with the UHF radar).

The solution is to use phase patterns of short pulses instead of spatial patterns. One of these techniques is the alternating code (Lehtinen and Häggström, 1987). It provides good range and lag resolution and has been proven to give near optimum accuracy in the case of poor signalto-noise ratio. The principle is to transmit a set of phasecoded pulses and decode them in such a way that only part of the pulse (one baud) is contributing to the scattering. An important assumption for the code to work properly is that the plasma correlation function must remain stationary in the time needed for the whole set of scans to be transmitted. A similar technique, the coded long pulse (Sulzer, 1989), has been used recently at the Arecibo Observatory facility (Djuth et al., 1994). The results are promising, but the implementation of the technique for such very long codes (512 bits) requires dedicated hardware processing.

\section{Experimental setup}

We used the VHF EISCAT radar near Tromsø, Norway at daytime in June 1994. The radar cannot be steered south to be in a field-aligned position, thus the antenna was pointed vertically, which gives an angle of about $12^{\circ}$ to the magnetic field line at $250 \mathrm{~km}$. The experiment used a 32-bit strong condition alternating code of baud length $20 \mu$ s (actually the first alternating code run on the VHF system). Three receiving channels, one for upshifted, one for downshifted plasma lines, and one for the ion line, were used simultaneously with the same spatial characteristics, thus providing the two components of the incoherent scatter spectrum. Forty gates were formed from an altitude (or range) of $180 \mathrm{~km}$ up to $300 \mathrm{~km}$ with an altitude resolution of $3 \mathrm{~km}$. The gate separation was also $3 \mathrm{~km}$. The autocorrelation function was measured from a delay of $20-620 \mu \mathrm{s}$ in steps of $20 \mu \mathrm{s}$. This means a spectrum of $50-\mathrm{kHz}$ bandwidth and about $780-\mathrm{Hz}$ resolution. To get the zero-lag of the autocorrelation function, a 1baud-length pulse was also used with the same geometric characteristics. Each interpulse period was $\sim 13 \mathrm{~ms}$ and for a 32-bit alternating code one has to send 64 different coded long pulses, consequently the whole cycle was $\sim 0.8 \mathrm{~s}$. This is the time we have to assume that the ionosphere remains stationary. The data were dumped every $10 \mu$ s. This setup is a good compromise for a high- enough height resolution, a large enough bandwidth of the receiver, and the physical limitation of the size of the memory of the acquisition system. For example, if we assume a Chapman layer in the neighborhood of the peak of the $F$ region, with a characteristic scale height of $65 \mathrm{~km}$, and if the receiver system is tuned at the peak or shifted inside the peak, we can expect to get at least six gates with signal falling within the bandwidth of the filter (Fig. 1).

\section{Data analysis}

\subsection{Ion-line data}

The ion-line data were analyzed in 10-s intervals, providing the four standard ionospheric parameters, i.e., electron density, electron and ion temperature, as well as ion mean drift velocity along the line of sight. Figure 2 shows the results of the analysis. One can see that the ionosphere had a very quiet and steady behavior, as well as a rather steep electron temperature gradient.

\subsection{Power-profile data}

The plasma-line power profiles were analyzed solving Eq. 1 below (Yngvesson and Perkins, 1968), a similar
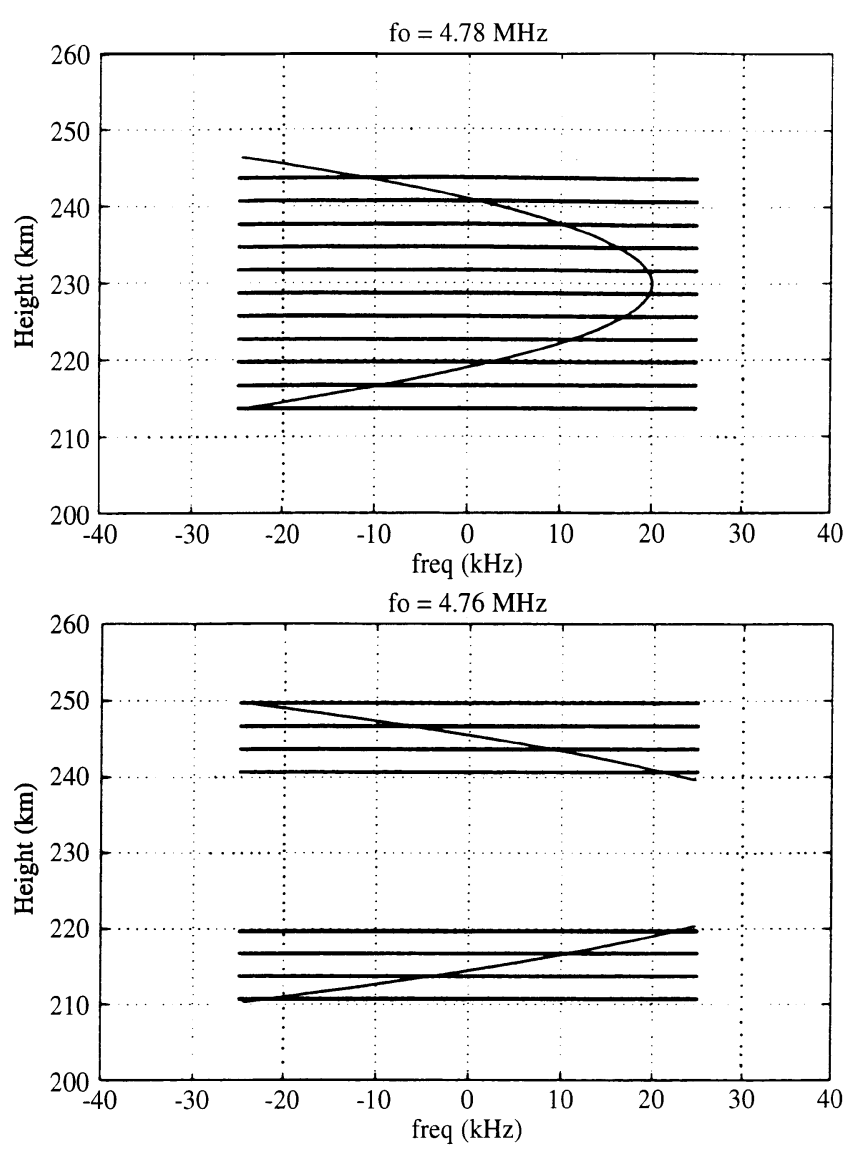

Fig. 1. Parabolic height distribution of the plasma-line frequency with scale height of $65 \mathrm{~km}$ showing two different tunings of the $50-\mathrm{kHz}$ receiving filter and the corresponding height cells contributing to the scattering 
ALT32V 1994-06-25

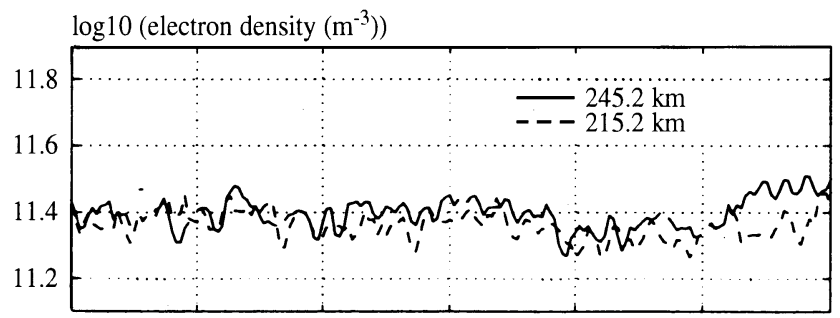

Electron temperature $(\mathrm{K})$
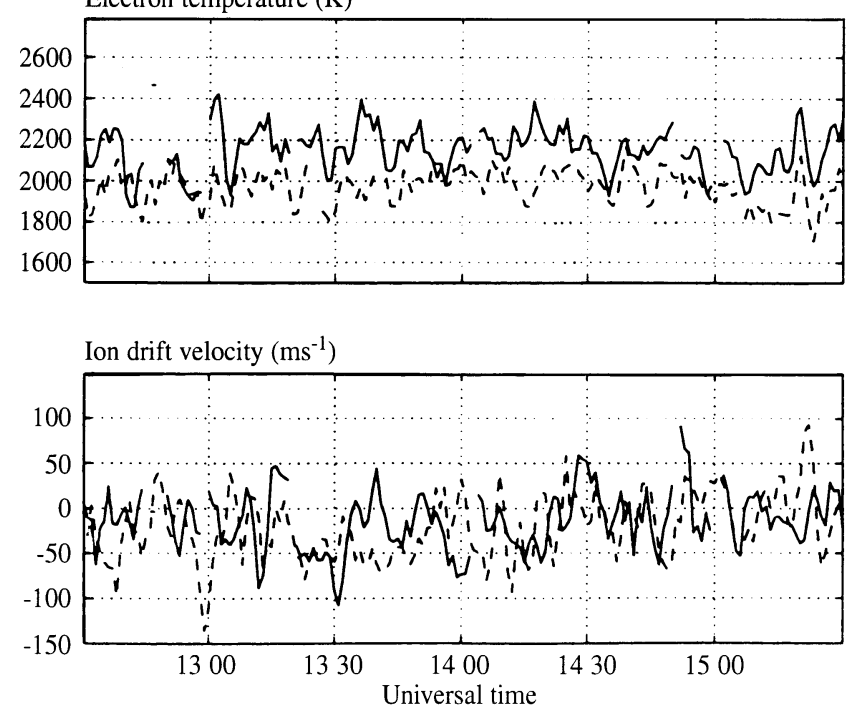

Fig. 2. Ionospheric parameters as function of range and time from the analysis of the ion line. The data have been smoothed with a $9 \mathrm{~km} \times 30 \mathrm{~s}$ window

radar equation as the one used for the ion line, yielding parameters $Q_{u}$ and $Q_{d}$ for the upshifted and downshifted lines (Fig. 3).

$Q=\frac{P_{R}}{P_{T}} \frac{R^{2}}{\delta R}\left(C_{s} \frac{A(f)}{A(0)}\right)^{-1}$.

Here, $P_{T}$ and $P_{R}$ denote transmitted and received power, $R$ is the distance to the probed volume, $\delta R$ is the volume extent (which is the minimum of the length of the transmitted pulse and the range with signal falling inside the receiver filter) and $C_{s}$ is the system constant. $A(f)$ is the frequency-dependent effective antenna area at frequency $f$ offset from the transmitted frequency. $A(f) / A(0)$ is the gain relative to the measurement of the ion line. It has been estimated for the VHF EISCAT system by measurement of the radio star Cassiopeia A.

The analysis shows a very similar enhancement of the upshifted and downshifted line, indicating the presence of isotropic photoelectrons. Situations where the receiving filter was tuned to a frequency not matching the frequency of the peak can be seen at different times on both lines, for example around 1245 and 1315 UT. Note also that the asymmetry after 1515 UT is artificial, and is produced by an increase in the receiver-filter bandwidth, thus increasing the noise level with aliased noise contributions.
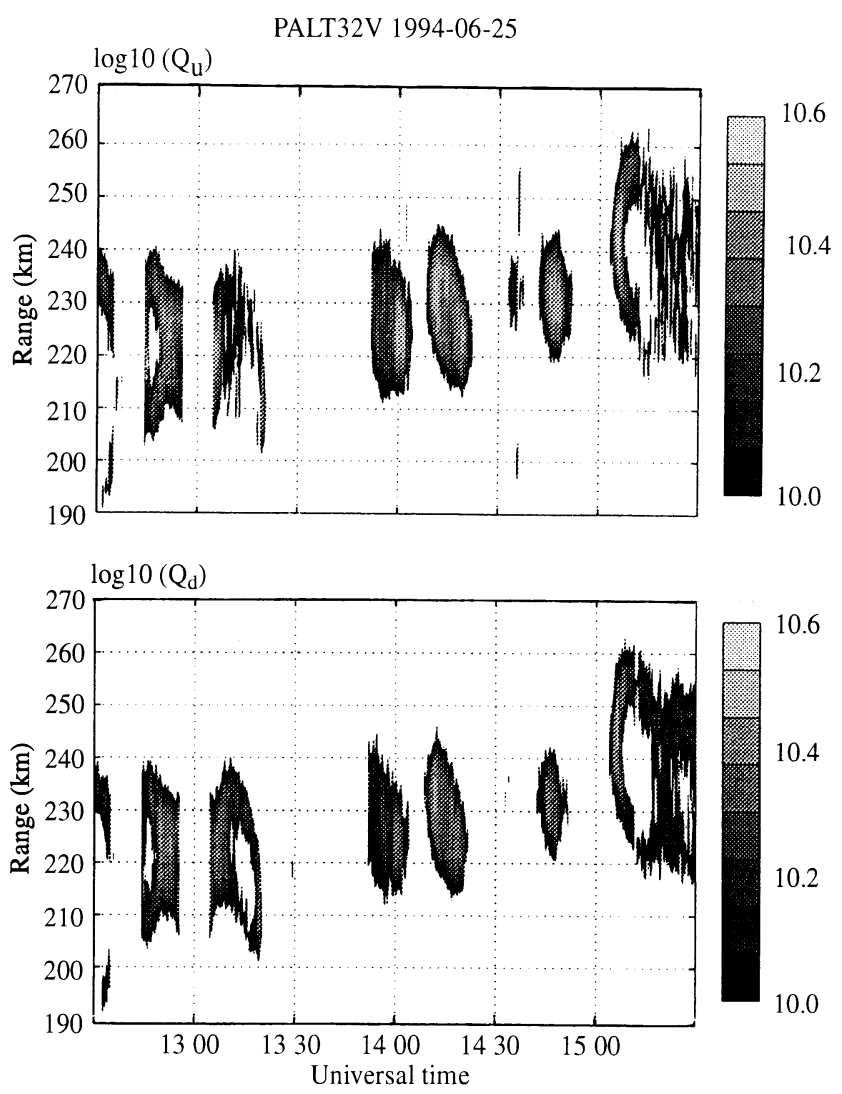

Fig. 3. The two parameters $Q_{u}$ and $Q_{d}$ of Eq. 1 as function of range and time from the analysis of the upshifted and downshifted plasmaline power profiles

\subsection{Autocorrelation-function data}

The autocorrelation functions were analyzed using a leastsquare fit method. We have tried two different spectral models, a squared-triangle shape and a Gaussian, both yielding three parameters: the received frequency $f_{r}$, the bandwidth of the line $\delta_{f}$, and the power of the line $a_{p}$. The model of the autocorrelation function $\rho$ for a set of parameters and at time delay $\tau$ is given by Eq. 2 for the squared- triangle model and Eq. 3 for the Gaussian model. The squared triangle is based on physical arguments: the amplitude sensitivity is maximum at the center of a range gate and falls linearly to zero one baud length away on each side (neglecting the effect of the receiver filter). As the plasma frequency is changing approximately linearly in a range gate, the returned power spectrum will take the shape of a squared triangle falling to zero at a certain frequency. It should be convolved with the natural-linewidth spectrum as well as multiplied by the receiver-filter response spectrum. The Gaussian shape is not rigorously true because it does not fall exactly to zero at a certain frequency, but it falls quickly enough toward zero to be justified. This spectral shape can be used to approximate a squared-triangle spectrum smoothed by the convolution effect of the natural line width. Figure 4 shows the results of both fit models for a 10-s dump. The curves are so similar that they are hardly separable from each other. In 


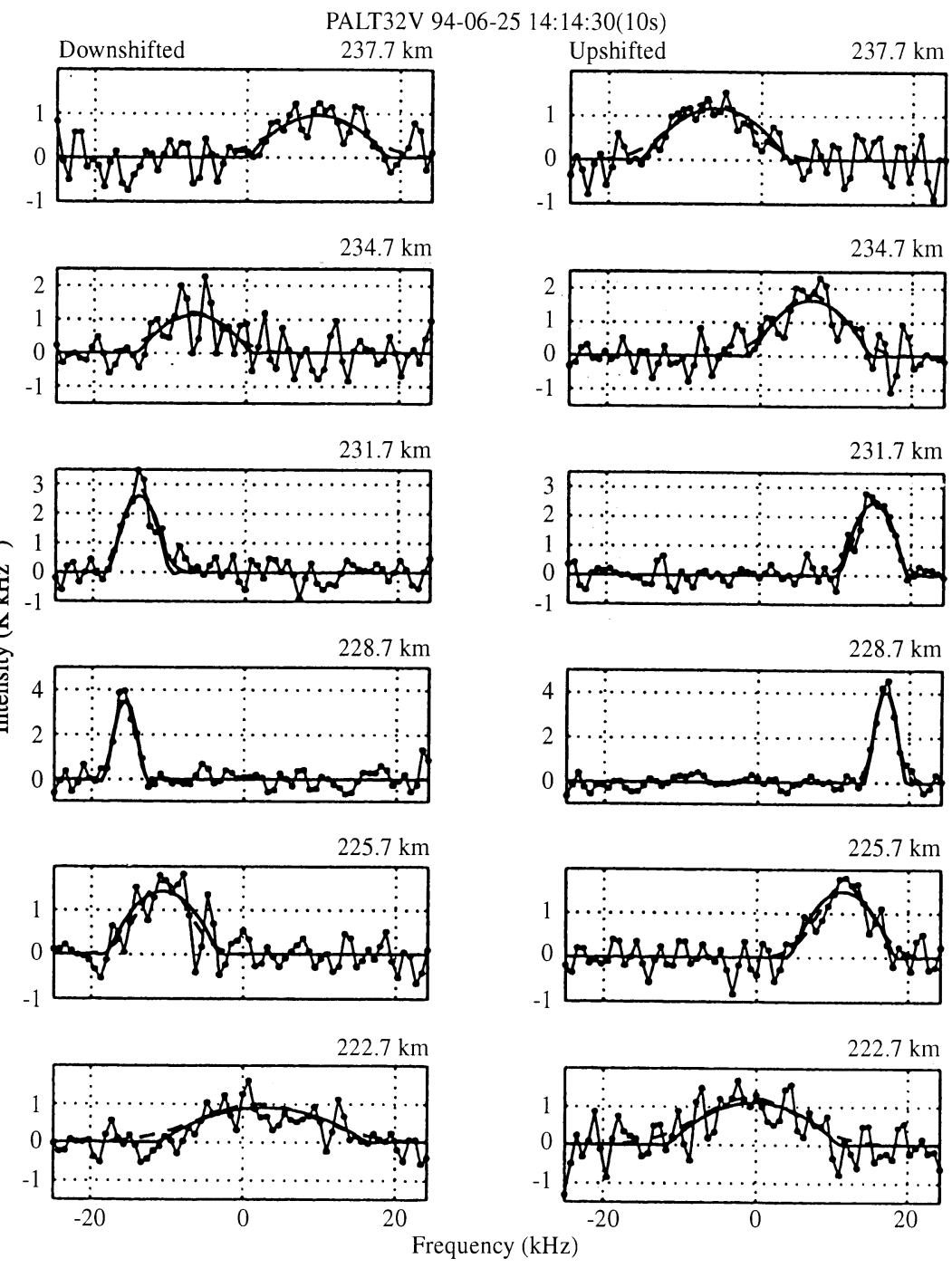

Fig. 4. Downshifted and upshifted spectra (circle points and thin solid line) from a 10-s dump fitted to the squared-triangle model (thick solid line) and the Gaussian model (dashed line). The scale is the same for each height

Fig. 5 the parameters of the fit models are plotted. All of the parameters exhibit a rather good agreement.

$$
\begin{aligned}
\rho_{S}\left(\tau ; f_{r}, \delta_{f}, a_{p}\right)= & 3 a_{p} \frac{\sin \left(4 \pi \delta_{f} \tau\right)-4 \pi \delta_{f} \tau \cos \left(4 \pi \delta_{f} \tau\right)}{\left(4 \pi \delta_{f} \tau\right)^{3}} \\
& \times \exp \left(i 2 \pi f_{r} \tau\right) \\
\rho_{G}\left(\tau ; f_{r}, \delta_{f}, a_{p}\right)= & a_{p} \exp \left(-\frac{\left(2 \pi \delta_{f} \tau\right)^{2}}{2}\right) \exp \left(i 2 \pi f_{r} \tau\right) .
\end{aligned}
$$

Figures 4 and 5 show the analysis of one data dump where six gates were fitted. Note that in Fig. 5 the received frequency $f_{r}$ for the downshifted line is plotted positive. One can also see that the parameters exhibit very smooth behavior and very small error bars, $\sim 0.01 \%$ for the received frequency.

Figures 6-8 show the result of the analysis of the autocorrelation functions of the plasma lines for the whole set of data in a similar way as for ion-line data, i.e., in time-range coordinates. One can see in Fig. 7 the general trend that the bandwidth of the line $\delta_{f}$ tends to increase as the position is moving away from a region of large scale height. This can also be seen in Fig. 6.

\section{Further analysis}

The solution of the real part of the dispersion relation for Langmuir waves gives the position of the frequency peak of the plasma-line spectrum. For a Maxwellian, homogeneous and unmagnetized electron plasma, this can be written as Eqs. 4 and 5 for the upshifted line (i.e., the downgoing Langmuir waves) and Eqs. 6 and 7 for the downshifted line (upgoing Langmuir wave).

$k_{+}^{2} \lambda_{D}^{2}+\mathrm{W}_{\mathrm{r}}\left(\frac{\omega_{r^{+}}-k_{+} V_{e}}{k_{+} v_{e}}\right)=0$

$k_{+}=\frac{2 \pi f_{\text {radar }}}{c}+\left(\frac{2 \pi f_{\text {radar }}}{c}+\frac{\omega_{r^{+}}}{c}\right)$

$k_{-}^{2} \lambda_{D}^{2}+\mathrm{W}_{\mathrm{r}}\left(\frac{\omega_{r^{-}}-k_{-} V_{e}}{k_{-} v_{e}}\right)=0$

$k_{-}=\frac{2 \pi f_{\text {radar }}}{c}+\left(\frac{2 \pi f_{\text {radar }}}{c}+\frac{\omega_{r^{-}}}{c}\right)$.

Here, $\omega_{r^{+}}, k_{+}$and $\omega_{r^{-}}, k_{-}$denote the frequency at the peak and the corresponding wave number of the upshifted and downshifted spectra, respectively. $V_{e}$ and $v_{e}$ are the 


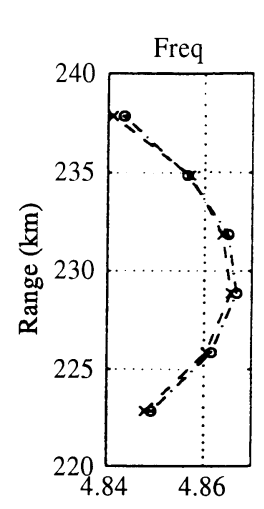

PALT32V 94-06-25 14:14:30(10s)
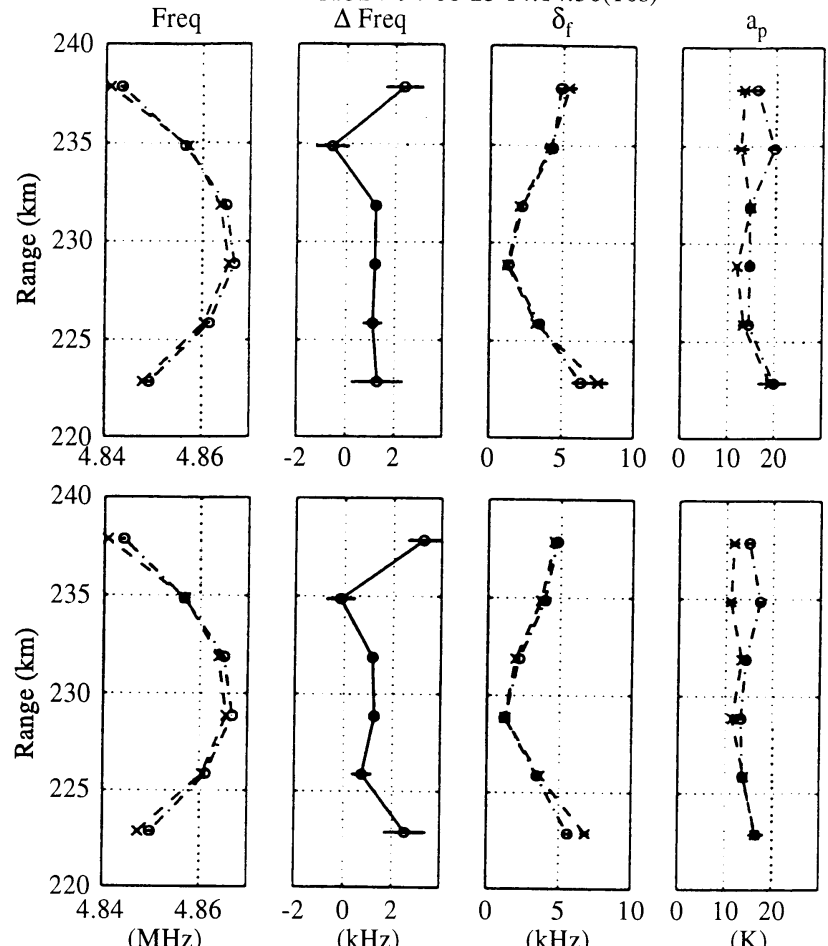

Fig. 5. The fitted parameters $f_{r}, \Delta f_{r}, \delta_{f}$ and $a_{p}$ as well as the error bars from the data of Fig. 4 as a function of range. The dashdot line is for the parameters from the upshifted line, and the dashed one is for the downshifted lines. The four upper panels show the parameters for the squared-triangle model, the four lower panels for the Gaussian model

electron drift velocity and the electron thermal velocity, $\lambda_{D}$ is the Debye length, and $\mathrm{W}_{\mathrm{r}}$ is the real part of the dispersion function W as defined by [Ichimaru (1973)].

By eliminating the Debye length $\lambda_{D}$, one can derive Eq. 8,

$k_{-}^{2} \mathrm{~W}_{\mathrm{r}}\left(\frac{\omega_{r^{+}}-k_{+} V_{e}}{k_{+} v_{e}}\right)=k_{+}^{2} \mathrm{~W}_{\mathrm{r}}\left(\frac{\omega_{r^{-}}-k_{-} V_{e}}{k_{-} v_{e}}\right)$.

This equation can be solved for $V_{e}$ when $\omega_{r^{+}}, k_{+}$and $\omega_{r^{-}}$, $k_{-}$are taken from the results of the plasma-line analysis and $v_{e}$ is calculated from the electron temperature given by the ion-line analysis.

When one wants to take into account the electron heat flow contribution introduced by Kofman et al. (1993), Eqs. 4 and 6 are modified to Eqs. 9 and 10, where $\mathscr{E}_{ \pm}$is the electron heat-flow correction.

$k_{+}^{2} \lambda_{D}^{2}\left(1-\mathscr{E}_{+}\right)+\mathrm{W}_{\mathrm{r}}\left(\frac{\omega_{\mathrm{r}^{+}}-k_{+} V_{e}}{k_{+} v_{e}}\right)=0$

with

$\mathscr{E}_{+}=\frac{8}{\left(\omega_{r^{+}}-k_{+} V_{e}\right)^{5}} \frac{k_{+}^{3} e^{2} q_{e} \cos \alpha}{m_{e}^{2} \varepsilon_{0}}$

$k_{-}^{2} \lambda_{\mathrm{D}}^{2}\left(1-\mathscr{E}_{-}\right)+\mathrm{W}_{\mathrm{r}}\left(\frac{\omega_{r^{-}}-k_{-} V_{e}}{k_{-} v_{e}}\right)=0$

with

$\mathscr{E}_{-}=\frac{8}{\left(\omega_{r^{-}}-k_{-} V_{e}\right)^{5}} \frac{k_{-}^{3} e^{2} q_{e} \cos \alpha}{m_{e}^{2} \varepsilon_{0}}$.
PALT32V 1994-06-25

Upshifted frequency $(\mathrm{MHz})$
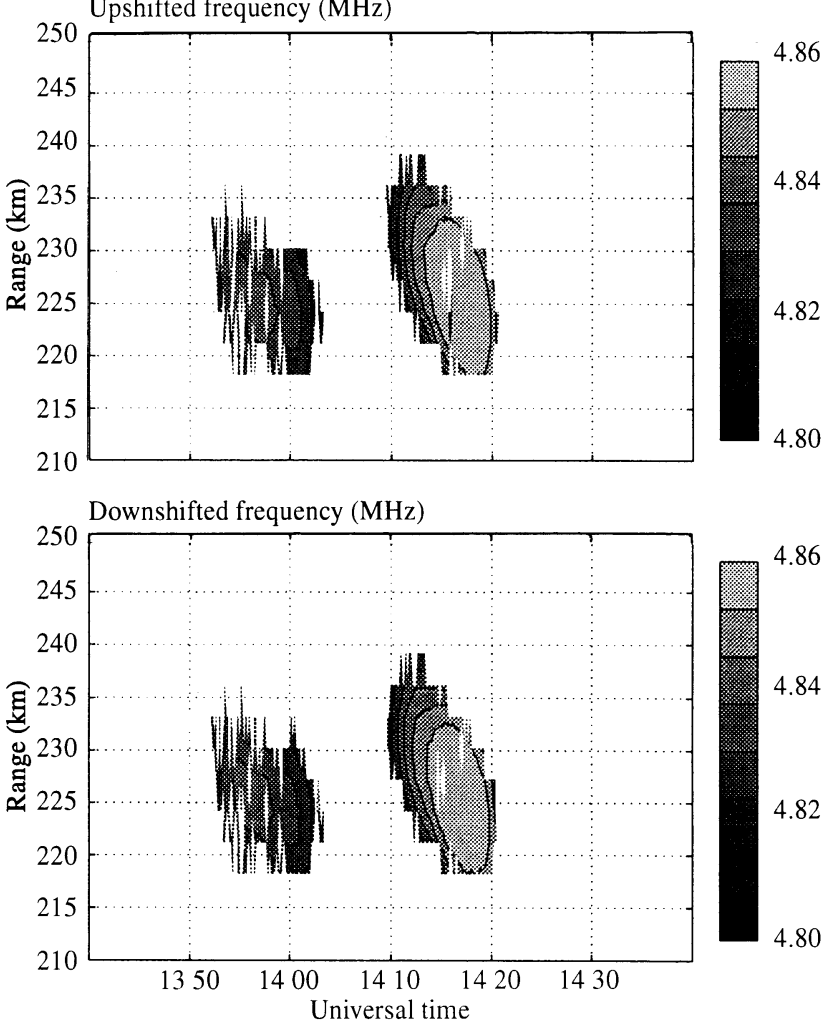

Fig. 6. The fitted upshifted and downshifted received frequencies displayed as a function of range and time for the Gaussian model
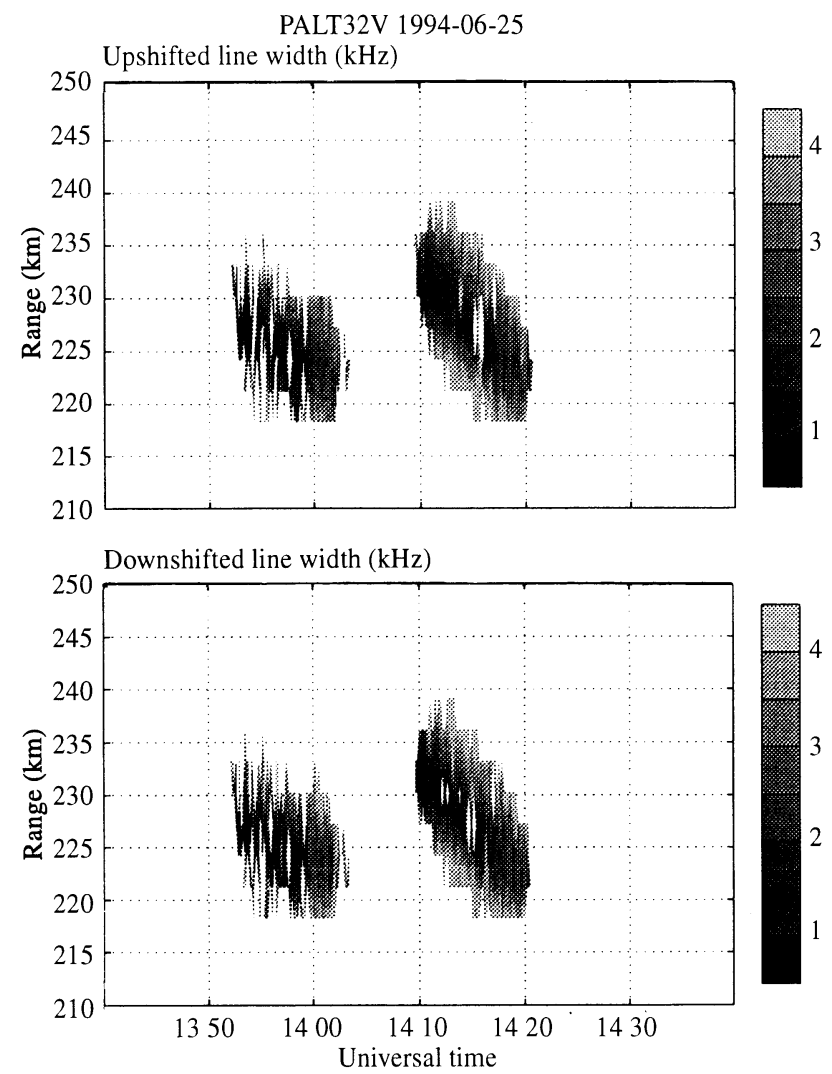

Fig. 7. The fitted upshifted and downshifted line bandwidth displayed as a function of range and time for the Gaussian model 


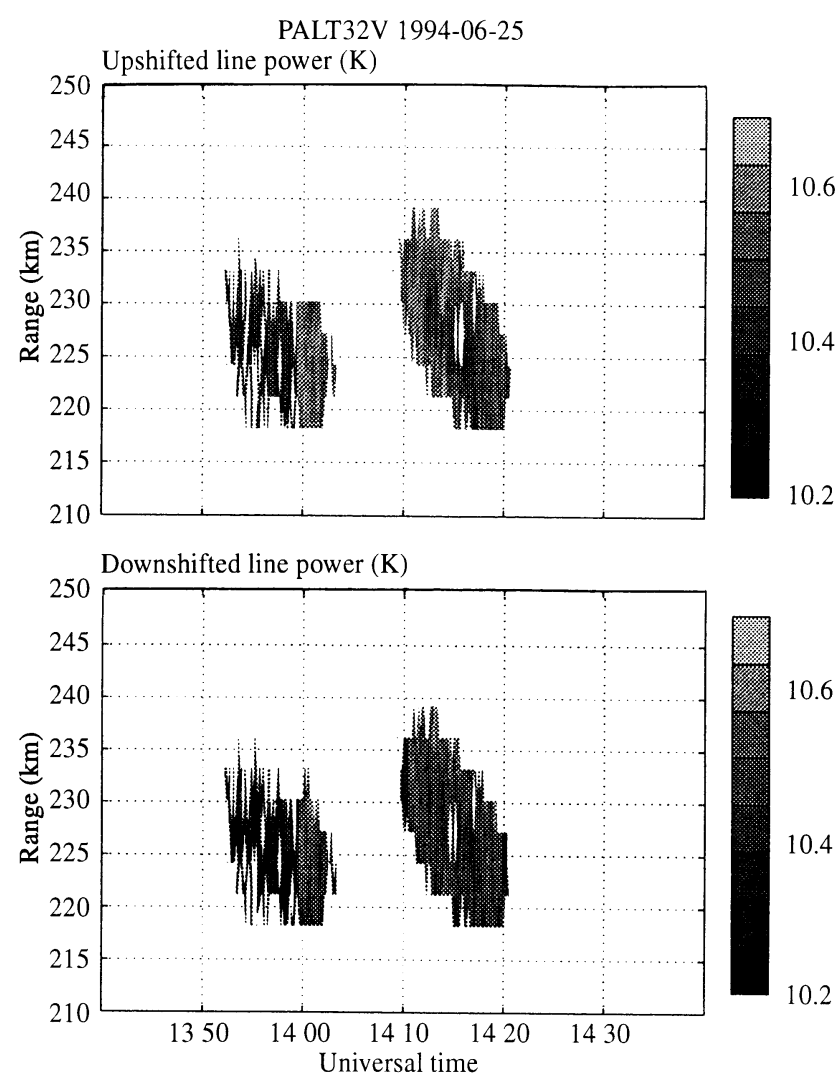

Fig. 8. The fitted upshifted and downshifted line power displayed as a function of range and time for the Gaussian model

This heat-flow correction is proportional to the third-order moment of the one-dimensional electron-velocity distribution function along the line of sight of the radar. It can be introduced in the dispersion relation by noting that when $\mathrm{W}_{\mathrm{r}}$ is expanded for waves with phase velocity much larger than the electron thermal velocity, as for Langmuir wave, it takes the form of a series of the successive moments of the electron-velocity distribution function. In the correction term $\mathscr{E}_{ \pm}, q_{e}$ is the heat flow along the magnetic field, $\alpha$ is the angle between the line of sight of the radar and the magnetic field, $m_{e}$ and $e$ are the mass and charge of the electron; $q_{e}$ is in the limit of a fully ionized gas a function of the local electron-temperature gradient. At ionospheric heights and along the magnetic field, it is given by (Banks, 1966)

$q_{e}=-1.2310^{-11} T_{e}^{5 / 2} \nabla T_{e} \quad \mathrm{~J} \mathrm{~m}^{-2} \mathrm{~s}^{-1}$.

In the same way as for Eqs. 4 and $6, \lambda_{D}$ can be eliminated from Eqs. 9 and 10, leading to Eq. 12

$$
\begin{aligned}
k_{-}^{2} & \left(1-\mathscr{E}_{-}\right) \mathrm{W}_{\mathrm{r}}\left(\frac{\omega_{r^{+}}-k_{+} V_{e}}{k_{+} v_{e}}\right) \\
= & k_{+}^{2}\left(1-\mathscr{E}_{+}\right) \mathrm{W}_{\mathrm{r}}\left(\frac{\omega_{r^{-}}-k_{-} V_{e}}{k_{-} v_{e}}\right) .
\end{aligned}
$$

This equation can also be solved for $V_{e}$ when $\omega_{r^{+}}$, $k_{+}$and $\omega_{r^{-}}, k_{-}$are taken from the results of the plasmaline analysis and $v_{e}$ is calculated from the electron temperature given by the ion-line analysis. Also, the electrontemperature gradient has to be estimated to calculate $\mathscr{E}_{ \pm}$.
To get the gradient we have calculated an average electron-temperature profile during the time of the experiment, fitted it to a polynomial and then calculated the derivative from this model profile. This is justified by the fact that the electron temperature appears to have a very smooth behavior during the whole experiment. Figure 9 shows the model used for the gradient. Another justification to use an average profile is that the use of an estimated gradient for each dump would lead to unnecessarily noisy gradient estimates, whereas our estimate for the gradient shows a rather smooth behavior. At the considered heights between 200 and $260 \mathrm{~km}$, the gradient was of the order of $5 \mathrm{~K} \mathrm{~km}^{-1}$.

We now have an estimate of $V_{e}$. By use of one of Eqs. 4 and 6 (or similarly Eqs. 9 and 10 for the heat-flow model), we can calculate the electron density $n_{e}$ to a much better accuracy than the one we can get from the ion line. We now have all the necessary quantities to calculate the currents with Eq. 13,

$\mathrm{J}=n_{e} e\left(V_{e}-V_{i}\right) \quad \mathrm{Am}^{-2}$,

where $V_{i}$ is taken from the results of the ion-line analysis.

Figure 10 shows the histograms of the estimated currents for both models, with and without the heat-flow term. These histograms exhibit a very good normal distribution of the values of the currents. For the expectation of
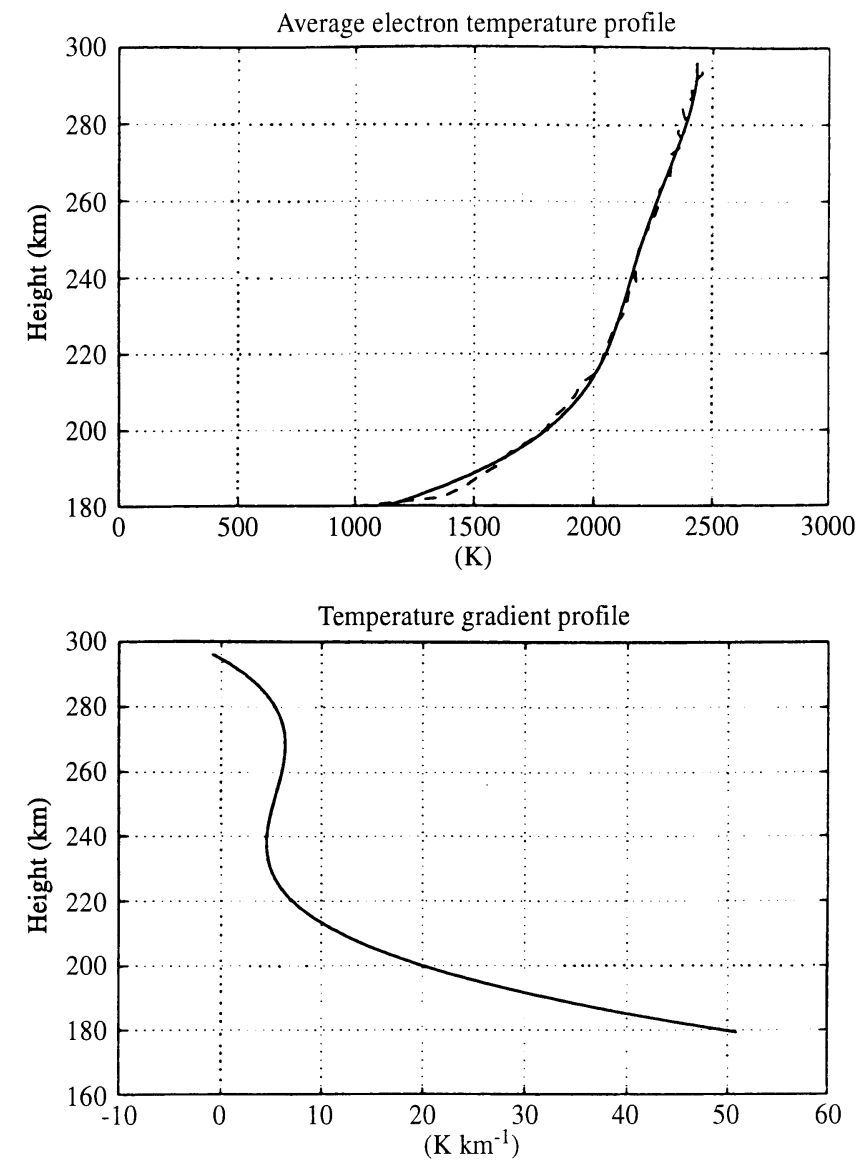

Fig. 9. Average electron-temperature profile for the considered period in dashed line and the polynomial approximation, and the deduced profile for temperature gradient 
a normal distribution, a 95\% confidence interval for the current $\mathbf{J}$ is given in Table 1 . It shows a good independence of the result from the fitting model.

One can see that the heat-flow correction is very effective in the estimation of the mean drift current in the case of large gradient temperature, and thus is in better agreement with no net parallel current during the quiet daytime conditions. Note also that the uncertainty on the estimated current remains of the same order; this is certainly to be accounted to the way we have estimated the electrontemperature gradient.
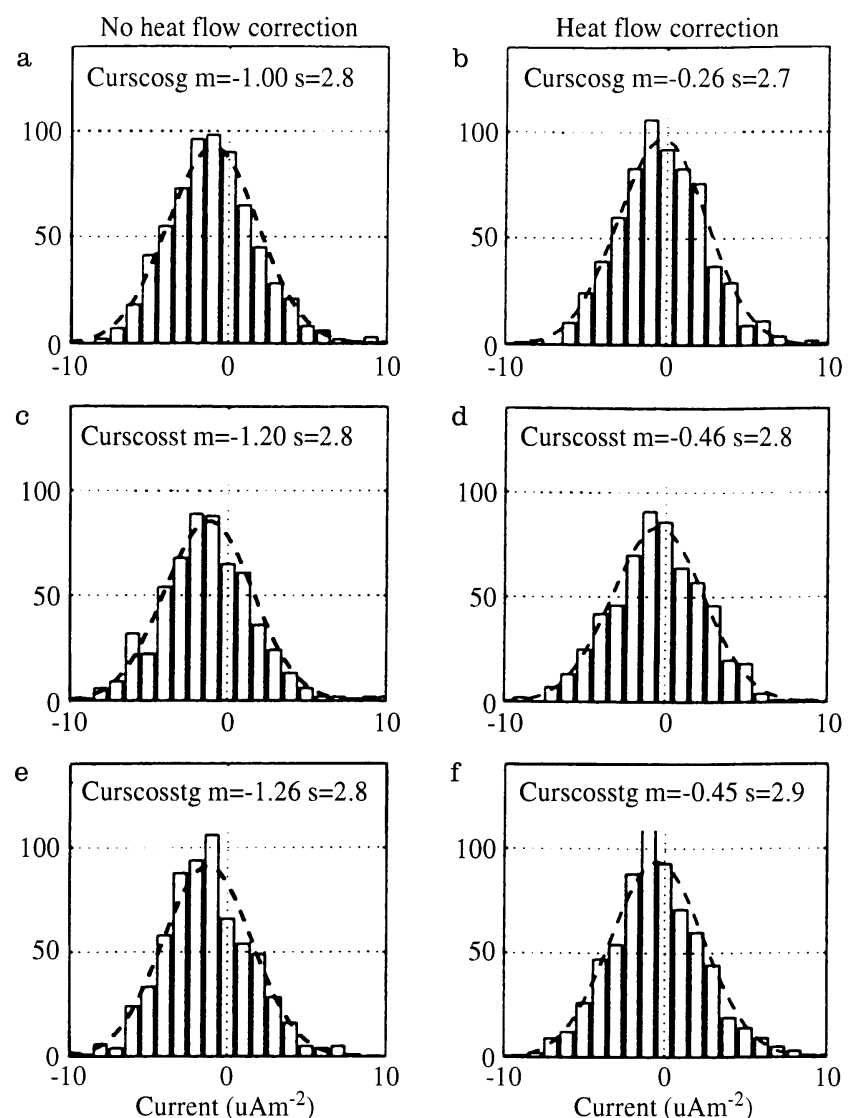

Fig. 10a-f. Histogram of the calculated currents without (left panels) and with heat-flow correction (right panels). The total population of each histogram is $\sim 700$. The dashed lines represent the best fit to a normal distribution. a and $\mathbf{b}$ are for the Gaussian model, $\mathbf{c}$ and $\mathbf{d}$ are for the squared-triangle model, and $\mathbf{e}$ and $\mathbf{f}$ are for a data analysis taking the parameters of the fit giving the smallest residuals of the squared-triangle and Gausian fits. Note that positive currents are upward currents

Table 1. $95 \%$ confidence interval for the currents $\mathrm{J}$ without heatflow correction and $\mathrm{J}_{\mathrm{hf}}$ with heat-flow correction from the histograms of Fig. 10

\begin{tabular}{lll}
\hline & $\mathrm{J}\left(\mu \mathrm{A} \mathrm{m}^{-2}\right)$ & $\mathrm{J}_{\mathrm{hf}}\left(\mu \mathrm{A} \mathrm{m}^{-2}\right)$ \\
\hline Gaussian model (a) (b) & $-1.0 \pm 0.2$ & $-0.3 \pm 0.2$ \\
Squared-triangle model (c) (d) & $-1.2 \pm 0.2$ & $-0.5 \pm 0.2$ \\
Best of both models (e) (f) & $-1.3 \pm 0.2$ & $-0.4 \pm 0.2$ \\
\hline
\end{tabular}

\section{Conclusion}

We have shown that the alternating code for studying plasma lines with an incoherent scatter radar is a valuable technique. It yields a greatly improved spatial resolution and a much larger amount of measured points (between five and six times more than for a long-pulse experiment).

We have been using our data to test the heat-flow dispersion-relation model under quiet and steady ionospheric conditions. We confirm that the use of the heatflow model gives important corrections and improves the measurements of current.

The plans are to extend and improve the use of the alternating code in future experiments. On one hand, the experiment could be refined and improved to give better accuracy and larger quantities of data. On the other, analysis techniques such as constrained full profile analysis of both plasma-line and ion-line data should be investigated and tried.

Acknwledgements. The authors thank one of the referees for his valuable remark about the spectral models for the fitting procedure. The authors want to thank the EISCAT personnel for their efforts in making the radar available. EISCAT is funded by the CNRS of France, the SA of Finland, the MPG of Germany, the NFR of Norway, the NFR of Sweden, and the PPARC of United Kingdom.

Topical Editor D. Alcaydé thanks M. Lehtinen and another referee for their help in evaluating this paper.

\section{References}

Banks, P. M., Charged particle temperatures and electron thermal conductivity in the upper atmosphere, Ann. Geophysicae, 22, 577-587, 1966.

Birkmayer, W., and T. Hagfors, Observational technique and parameter estimation in plasma line spectrum observations of the ionosphere by chirped incoherent scatter radar, J. Atmos. Terr. Phys., 48, 1009-1019, 1986.

Djuth, F. T., M. P. Sulzer, and J. H. Elder, Application of the coded long-pulse technique to plasma line studies of the ionosphere, Geophys. Res. Lett., 21, 2725-2728, 1994.

Farley, D. T., Multiple-pulse incoherent-scatter correlation function measurements, Radio Sci., 7, 661-666, 1972.

Hagfors, T., Incoherent scatter radar observations of the plasma line with a chirped pulse system, Radio Sci., 17, 727-734, 1982.

Ichimaru S., Basic principles of plasma physics, W. A. Benjamin, Reading, Mass., 1973.

Kofman, W., and V. Wickwar, Plasma line measurements at Chatanika with high-speed correlator and filter bank, J. Geophys. Res., 85, 2998-3012, 1980.

Kofman, W., J.-P. St-Maurice, and A.P. van Eyken, Heat flow effect on the plasma line frequency, J. Geophys. Res., 98, 6079-6085, 1993.

Lehtinen, M. S., and I. Häggström, A new modulation principle for incoherent scatter measurements, Radio Sci., 22, 625-634, 1987.

Perkins, F., E. E. Salpeter, Enhancement of plasma density fluctuations by nomthermal electrons, Phys. Rev. A, 139, 55-62, 1965.

Showen, R. L., The spectral measurement of plasma line, Radio Sci., 14, 503-508, 1979.

Sulzer, M. P., Recent incoherent scatter techniques, Adv. Space Res., 9, 153-161, 1989.

Yngvesson, K. O., and F. W. Perkins, Radar Thomson scatter studies of photoelectrons in the ionosphere and Landau damping, $J$. Geophys. Res., 73, 97-110, 1968.

This article was processessed by the author using the $\mathrm{L}^{\mathrm{A}} \mathrm{T} \mathrm{X}$ style file pljour2 from Springer-Verlag. 